\title{
Radiation doses to emergency department patients undergoing computed tomography
}

\author{
James C. Worrall, MD*; Sadia Jama, BSc*; Ian G. Stiell, MD, MSc* ${ }^{* \ddagger}$
}

\section{ABSTRACT}

Objectives: Computed tomography (CT) use is increasing in the emergency department (ED). Many physicians are concerned about exposing patients to radiation from CT scanning, but estimates of radiation doses vary. This study's objective was to determine the radiation doses from CT scanning for common indications in a Canadian ED using modern multidetector CT scanners.

Methods: We conducted a health records review of consecutive adult patients seen at two busy tertiary care EDs over a 2month period who underwent CT scanning ordered by emergency physicians. Cases were identified by searching an imaging database. Data collected included patient age and sex, study indication, scanner model, body area, and reported dose-length product. Effective dose per scan was calculated from reported dose-length product. Data were collected on a standardized form, entered into an electronic database, and analyzed with descriptive statistics and $95 \% \mathrm{Cls}$.

Results: During the study period, emergency physicians assessed 19,880 patients. Overall, 2,720 (13.7\%) underwent CT scanning, and of these, 144 (5.3\%) patients had more than one scan. Patients had a mean age of 59.0 years, and $45.3 \%$ were men. Mean doses for the most common indications were as follows: simple head, $2.9 \mathrm{mSv}$; cervical spine, $5.7 \mathrm{mSv}$; complex head, $9.3 \mathrm{mSv}$; CT pulmonary angiogram, $11.2 \mathrm{mSv}$; abdomen (nontraumatic abdominal pain), $15.4 \mathrm{mSv}$; and abdomen (renal colic), $9.8 \mathrm{mSv}$.

Conclusions: Approximately one in seven ED patients had a CT scan. Emergency physicians should be aware of typical radiation doses for the studies they order and how the dose varies by protocol and indication.

\section{RÉSUMÉ}

Objectif: Le recours à la tomodensitométrie (TDM) est à la hausse dans les services d'urgence (SU), et l'exposition des patients au rayonnement des appareils de TDM préoccupe bon nombre de médecins, mais les estimations des doses sont variables. L'étude visait à déterminer les doses de rayonnement émises par des appareils modernes de TDM à détecteurs multiples, utilisés dans un hôpital, au Canada, dans des indications courantes.

Méthode: Nous avons procédé à un examen des dossiers médicaux d'adultes consécutifs, qui ont été examinés dans deux SU de soins tertiaires, ayant une forte fréquentation, sur une période de 2 mois, et qui ont subi un examen par TDM à la demande d'urgentologues. La recherche de cas s'est faite dans une base de données d'images. La collecte de données comprenait l'âge et le sexe des patients, les indications de l'examen, le modèle d'appareil, la région du corps, ainsi que le produit dose-longueur indiqué. La dose efficace par balayage a été calculée à l'aide de ce produit dose-longueur. Les données ont été recueillies sur un formulaire normalisé, inscrites dans une base de données électroniques, puis analysées à l'aide de statistiques descriptives et d'intervalles de confiance à $95 \%$.

Résultats: Durant la période à l'étude, 19880 patients ont été examinés par des urgentologues; 2720 (13.7\%) d'entre eux ont subi une TDM, et sur ce nombre, 144 (5.3\%) ont été soumis à plus d'un balayage. L'âge moyen était de 59.0 ans, et $45.3 \%$ des malades étaient des hommes. Les doses moyennes de rayonnement dans les indications les plus fréquentes s'établissaient comme suit: examen simple de la tête, $2.9 \mathrm{mSv}$ (millisievert); colonne cervicale, $5.7 \mathrm{mSv}$; examen composé de la tête, $9.3 \mathrm{mSv}$; angiographie pulmonaire par TDM, $11.2 \mathrm{mSv}$; abdomen (douleur abdominale, non traumatique), $15.4 \mathrm{mSv}$; et abdomen (colique néphrétique), $9.8 \mathrm{mSv}$.

Conclusions: Environ un patient sur sept a été soumis à un examen par TDM au SU. Les urgentologues devraient être au courant des doses normales de rayonnement liées aux examens prescrits et ainsi que de la variation des doses selon les protocoles et les indications.

Keywords: computed tomography, emergency department, health records, radiation

From the Departments of *Emergency Medicine and †Epidemiology and Community Medicine, University of Ottawa; and $\ddagger$ Ottawa Hospital Research Institute, Ottawa, ON.

Presented at the Canadian Association of Emergency Physicians' Annual Scientific Assembly, St. John's, NL, 2011.

Correspondence to: Dr. James Worrall, The Ottawa Hospital, Civic Campus, 1053 Carling Avenue, 6th Floor, Room F662, Ottawa, ON K1Y 4E9; jaworrall@toh.on.ca.

This article has been peer reviewed.

(C) Canadian Association of Emergency Physicians 
Computed tomography (CT) is a commonly used diagnostic test in the emergency department (ED). In 2007, 13.9\% of ED patients in the United States underwent a CT scan. ${ }^{1}$ Compared to 1996, this represented a 330\% increase. Physicians have been warned of the risks of radiation associated with increasing CT use. ${ }^{2}$ Indeed, it has been estimated that at current rates, CT scanning will ultimately cause 29,000 cancers annually in the United States, or $2 \%$ of total cancers. ${ }^{3}$

Unfortunately, physicians may not fully understand how CT scanning affects a patient's future risk of cancer. ${ }^{4}$ Published estimates of CT radiation dose vary considerably. Several national surveys of CT use in the United Kingdom report doses on older CT scanners, mainly single-slice CT. ${ }^{5}$ Newer CT scanners use multiple detectors and have faster scanning times, allowing advanced applications, such as CT angiography. The dose of any given CT study is not constant and may vary significantly between patients. ${ }^{6}$

Previous surveys of radiation dose have not been limited to ED patients; therefore, reported doses may not be typical of scans ordered for emergency indications. Also, emergency physicians may have poor knowledge of the radiation doses associated with the CT scans they order. ${ }^{7}$ The purpose of this study was to determine the doses from CT scanning for common indications in a large Canadian ED using modern multidetector CT scanners.

\section{METHODS}

We undertook a health records review of consecutive adult patients who had undergone CT scanning ordered by emergency physicians over a 2-month period (February and March 2010) and were seen at the two EDs of The Ottawa Hospital (the Civic and General campuses), both busy academic tertiary care teaching facilities. Cases were identified by searching our picture archiving and communications system (PACS) database. We restricted the search by type of study (CT), name of the ordering physician, and date.

A standardized form was used. The data collectors were two of the authors (J.C.W., S.J.). The data collected were patient age and sex, study indication, scanner model, body area, and reported dose-length product. This study was approved by the Research Ethics Board of The Ottawa Hospital.
CT studies were classified into 1 of 18 possible indications, as given in Table 1. The data collector determined the indication by reading the handwritten CT requisition, which is scanned and appended to the PACS file at The Ottawa Hospital. When there was ambiguity about classification, the principal investigator (J.C.W.) reviewed the requisition and determined the most appropriate class.

A CT scan may be repeated with and without contrast or timed to visualize contrast in first the arterial circulation and then the venous circulation. These studies are referred to as multiphase and were considered a single study for dose purposes, assuming that they occurred at the same time. If the patient was recalled later in the visit for a repeat study, the studies were considered separate (e.g., if unenhanced head CT was repeated later in the day, but with contrast, the doses were considered separate).

All data were entered into a Microsoft Excel database. Once the database was created, the following records were rechecked by the lead investigator (J.C.W.) to ensure correct dose-length products and study classification: all patient records for patients who had multiple CT scans; all patients who appeared in the database on more than one occasion; all vascular studies (complex head, neck [vascular], and aorta); and all abdominal (flank pain) studies. The dose of a CT scan was considered to be its effective dose, a product of the dose-length product and an anatomic conversion factor. This method of dose estimation and the derivation of the conversion factors are described in the literature. ${ }^{8,9}$ The conversion factors used are given in Table 2. The data on the number of hospital visits were obtained from our administrative records. The data were analyzed using basic descriptive statistics. The types of CT scanners available at our institution are given in Table 3 . The majority of scans were performed on 64-slice CT scanners.

We reviewed the radiology reports of two subsets of patients: those undergoing CT thorax (pulmonary embolism $[\mathrm{PE}]$ protocol) and those undergoing cervical spine CT. We recorded the number of patients with positive findings ( $\mathrm{PE}$ or fracture), as well as other significant acute findings.

\section{RESULTS}

During the study period, there were 19,880 ED visits and emergency physicians ordered CT scanning in 


\section{Table 1. Types of CT scans ordered and associated radiation doses}

\begin{tabular}{|c|c|c|c|c|}
\hline Indication & Number of scans & Mean dose (mSv) & Median dose (mSv) & Interquartile range (mSv) \\
\hline Simple head & 1,543 & 2.9 & 2.5 & $2.3-3.3$ \\
\hline Abdomen (nontraumatic abdominal pain) & 555 & 15.4 & 12.3 & $9.9-18.8$ \\
\hline Thorax (PE protocol) & 186 & 11.2 & 10.2 & $9.6-11.4$ \\
\hline Abdomen (flank pain)* & 181 & 9.8 & 6.2 & $5.1-13.8$ \\
\hline Cervical spine & 91 & 5.7 & 5.2 & $4.4-6.3$ \\
\hline Neck (vascular) $^{\dagger}$ & 62 & 5.6 & 5.5 & $2.5-6.8$ \\
\hline Complex head ${ }^{\ddagger}$ & 50 & 9.3 & 8.8 & $7.9-10.5$ \\
\hline Lumbar spine & 29 & 17.7 & 17.3 & $10.7-24.6$ \\
\hline Facial bones ${ }^{\S}$ & 28 & 3.3 & 3.1 & $2.3-4.9$ \\
\hline Abdomen (trauma) & 23 & 13.6 & 10.9 & $9.3-17.9$ \\
\hline Thorax (trauma) & 19 & 11.7 & 7.5 & $6.9-14.7$ \\
\hline Aortic dissection protocol & 19 & 38.4 & 34.8 & $25.3-45.5$ \\
\hline Extremity & 15 & & & \\
\hline Neck (soft tissues) & 11 & 5.3 & 5.2 & $4.6-6.2$ \\
\hline Abdomen (aorta) $)^{\|}$ & 11 & 18.2 & 17.5 & $9.8-25.1$ \\
\hline Thoracic spine & 9 & 17.0 & 10.6 & $9.2-23.8$ \\
\hline Cardiac (CT coronary angiogram) & 8 & 22.7 & 25.6 & $23.4-28.4$ \\
\hline Thorax (aorta) & 2 & & & \\
\hline Other & 63 & & & \\
\hline \multicolumn{5}{|c|}{$\begin{array}{l}\text { CT = computed tomographic; PE = pulmonary embolism. } \\
\text { "An abdomen CT scan was classified as a flank pain study if the sole question was related to urolithiasis or renal pathology. If renal colic was listed as one of a number of possible diagnoses, } \\
\text { the study was classified as "abdomen (nontraumatic abdominal pain)." } \\
\text { 'This includes some patients who are also included under "complex head." Doses reported here represent the angiogram component. } \\
\text { FComplex head was defined as any study of the brain that included an angiogram or perfusion component. } \\
\text { sFacial bones was defined as any study where the physician queried facial fractures, regardless of whether or not the brain was imaged. } \\
\text { "An abdomen CT scan was classified as an aorta study if the sole question related to an abdominal aortic aneurysm (AAA) and the aortic dissection protocol was not used. If AAA was listed } \\
\text { as one of a number of possible diagnoses, the study was classified as "abdomen (nontraumatic abdominal pain)." }\end{array}$} \\
\hline
\end{tabular}

$2,720(13.7 \%)$ of these visits. These patients had a total of 2,883 scans as $144(5.3 \%)$ patients had more than one scan during their visit. Women comprised $54.7 \%$ of patients who underwent scanning. Nine scans had missing dose information on PACS. The characteristics of patients scanned are given in Table 3 and Figure 1. Patients age 35 or younger, who would be at higher risk from radiation exposure, comprised $15.3 \%$ of the patients scanned but $33 \%$ of the total ED population. The EDs studied see very few pediatric patients; only 5

\begin{tabular}{l}
$\begin{array}{l}\text { Table 2. Dose-length product to effective dose conversion } \\
\text { factors used }\end{array}$ \\
\hline Body area \\
Head
\end{tabular}

patients under 18 years of age had CT scans. The youngest was age 14 .

The numbers of each type of scan are reported in Table 1 . The most common CT scans ordered by

\begin{tabular}{|c|c|}
\hline Characteristic & $n(\%)$ \\
\hline \multicolumn{2}{|l|}{ Age (yr) } \\
\hline Mean & 59.0 \\
\hline Range & 14-102 \\
\hline$<35$ & $416(15.2)$ \\
\hline $36-55$ & $776(28.5)$ \\
\hline $56-75$ & $790(29.1)$ \\
\hline$>75$ & $737(27.1)$ \\
\hline Sex, male & $1,233(45.3)$ \\
\hline \multicolumn{2}{|l|}{ Scanner used } \\
\hline GE LightSpeed VCT (64 slice) & $1,467(53.9)$ \\
\hline Toshiba Aquilion 64 (64 slice) & $1,054(38.7)$ \\
\hline GE LightSpeed Plus (4 slice) & $163(6.0)$ \\
\hline Toshiba Aquilion One & $34(1.2)$ \\
\hline GE LightSpeed 16 & $5(0.2)$ \\
\hline
\end{tabular}




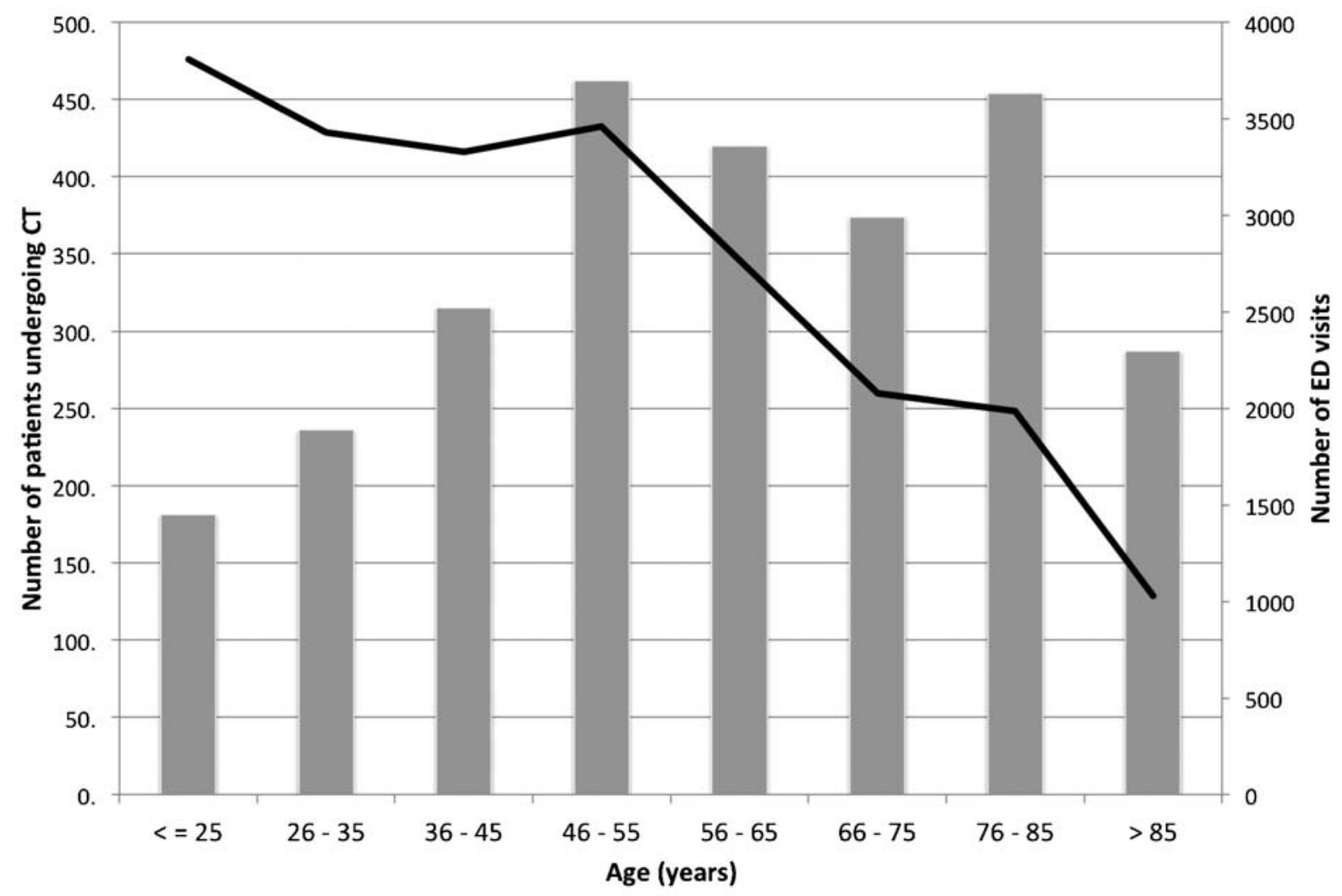

- Patients undergoing CT (bar) - Number of ED visits (line)

Figure 1. Age of patients undergoing computed tomography (CT). ED = emergency department.

emergency physicians were for the head, abdomen (for abdominal or flank pain), and thorax (for PE). Some scan types showed substantial variation in dose. High doses were noted for the aortic dissection protocol and the CT coronary angiogram.

Dose distributions showed positive (rightwards) skew for all study types except CT coronary angiograms, implying that a subset of patients receive doses substantially higher than the median. For the four most frequently ordered types of CT scan, the distributions of doses are shown in Figure 2. We noted considerable variation in doses, as evidenced by wide interquartile ranges, for the abdominal CT scans. Among the 1,531 simple head CT scans, only $2.3 \%$ of doses were more than twice the median dose. For thorax CT scans (PE protocol), only $4.3 \%$ were twice the median, whereas for abdominal CT scans for nontraumatic abdominal pain and flank pain, these figures were $13.3 \%$ and $31.5 \%$, respectively. We did not note consistently higher doses for any given scanner model.

Table 4 describes the findings in the two subsets of CT scans for which we estimated the overall "rule in" or diagnostic yield rate of the studies ordered. PE was diagnosed in $16 \%$ of patients who underwent thorax CT (PE protocol), and there were other findings identified in another $25 \%$ of patients. Cervical spine CT demonstrated acute findings in 8 of 92 patients $(9 \%)$.

\section{DISCUSSION}

This study is, to our knowledge, the first assessment of general CT use in a Canadian ED setting. We found that a high proportion of ED patients at our institutions underwent CT scanning. We also found that there is considerable variation in radiation dose for any type of CT scan, although this variation is greatest for abdominal scans.

Two recent American studies have described increasing use of CT imaging in EDs. ${ }^{1,10}$ High rates of CT use may lead to increases the in cost and risk of cancer. ${ }^{11}$ Although most patients who undergo CT have only a small associated lifetime attributable risk of cancer, a subset of patients are at higher risk. In one 

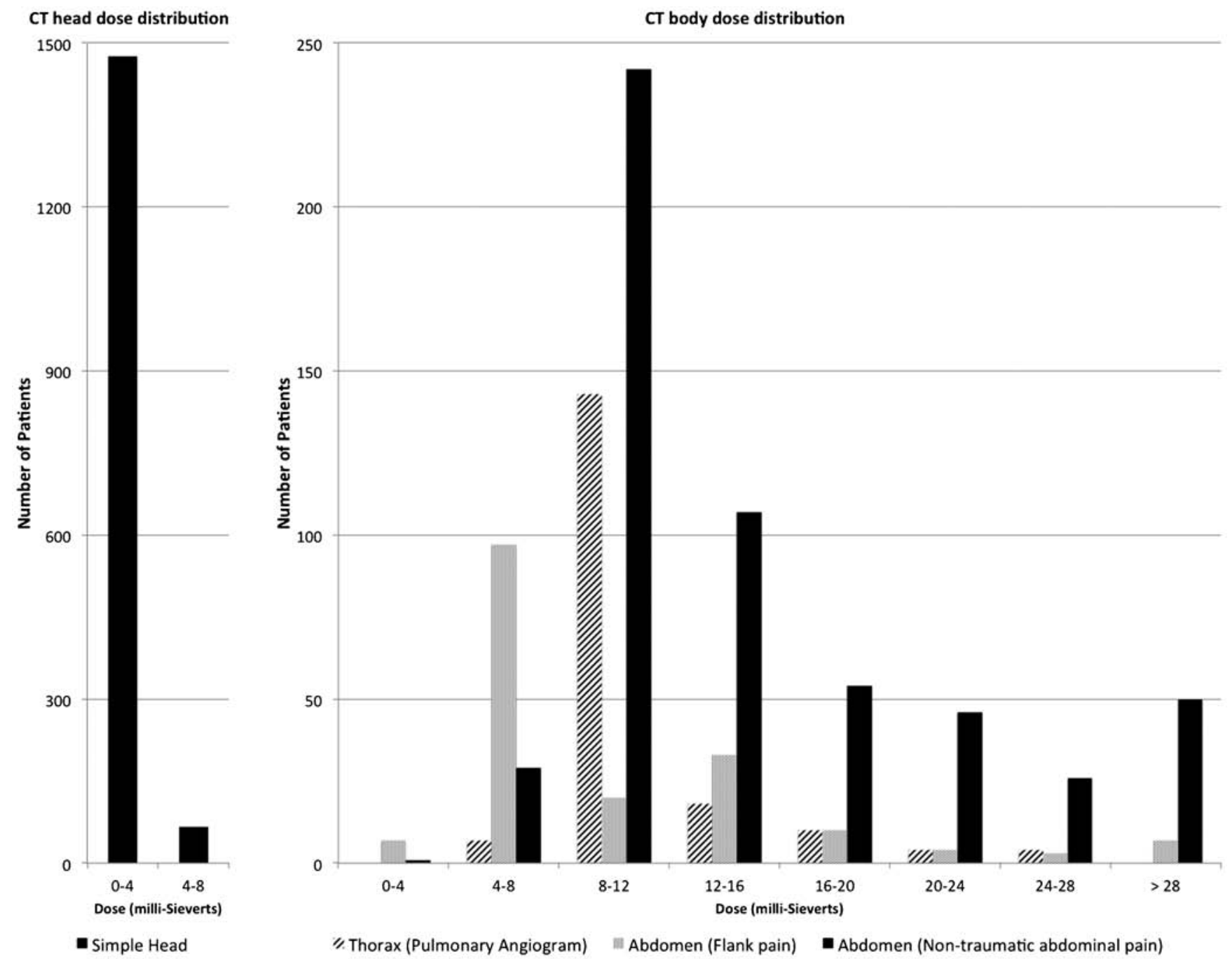

Figure 2. Distribution of radiation doses for the most common computed tomographic scans.

large cohort, $15 \%$ of patients received lifetime radiation doses greater than $100 \mathrm{mSv}$ and $7 \%$ had a lifetime attributable risk of cancer greater than 1 in $100 .{ }^{12}$ Another large study found that $1.9 \%$ of patients received more than $20 \mathrm{mSv}$ per year over 3 years. ${ }^{13}$

Unfortunately, it is not clear that increased CT use leads to improved patient outcomes. In our study, CT scans were commonly ordered by emergency physicians in cases of abdominal pain, flank pain, or suspected PE. Other studies suggest that routine abdominal CT scans in cases of suspected appendicitis may not reduce the negative appendectomy rate. ${ }^{14-16} \mathrm{In}$ cases of suspected renal colic, CT scans are frequently ordered, and many patients undergo multiple scans, but few patients receive urgent urologic intervention. ${ }^{17,18}$ CT pulmonary angiography is frequently ordered, possibly because of increased use of D-dimer testing, but mortality from PE diagnosed in US EDs is only $1 \% .{ }^{19,20}$ A recent multicentre study found that emergency physicians overuse CT in the investigation of $\mathrm{PE}$ and image more than half of low-risk patients. ${ }^{21}$ One-third of ED patients evaluated for PE will later be re-evaluated with a second CT scan, despite limited diagnostic yield. ${ }^{22}$ In our study, one in six patients scanned had a positive CT scan for PE, a rate comparable to that of a published trial of high-risk patients. $^{23}$

It is also likely that some CT scans are ordered without good clinical indication. ${ }^{24}$ Clinical decision rules might be useful to reduce unnecessary imaging, but one study demonstrated an increase in CT imaging despite implementation of the Canadian CT Head Rule. ${ }^{25}$ Furthermore, it is possible that clinical practice guidelines may encourage increased use of CT imaging. ${ }^{26}$ Given the cost, radiation risk, and lack of clear evidence of improved outcomes, it is debatable 


\begin{tabular}{ll}
\hline Table 4. Results of selected computed tomographic scans \\
\hline Result & $n(\%)$ \\
\hline Thorax (PE protocol) & \\
No & 189 \\
Normal & $95(50)$ \\
Pulmonary embolism & $31(16)$ \\
$\quad$ Subsegmental & 2 \\
Segmental & 19 \\
Lobar or central artery & 10 \\
Other & $63(33)$ \\
$\quad$ Suboptimal study to exclude PE & 21 \\
Neoplasm (new/progression) & 16 \\
Infectious/inflammatory & 12 \\
Pulmonary edema & 10 \\
Other & 12 \\
Cervical spine & \\
No & 92 \\
No fracture/acute finding & $84(91)$ \\
Fracture & $4(4)$ \\
Acute disk/ligamentous finding & $4(4)$ \\
\hline PE = pulmonary embolism. & \\
\hline
\end{tabular}

whether the increasing use of CT scanning by emergency physicians is justifiable.

The doses observed in this study are higher than those in an older British national survey of CT use, ${ }^{5}$ but they are similar to those in a recent four-hospital study in San Francisco. ${ }^{6}$ Like the latter study, we found significant variation in dose among studies of the same type. Sources of this dose variation are not fully clear. One likely factor is automatic exposure control algorithms on modern scanners, which adjust CT parameters to keep constant the noise in the CT image. ${ }^{27}$ For larger or obese patients, this means that the CT scanner will automatically increase the $x$-ray tube current to compensate for attenuation of the $\mathrm{x}$-ray beam by body tissue. One study found that increasing patient weight from 60 to $100 \mathrm{~kg}$ led to a tripling of reported dose-length product, although the estimated organ doses were only doubled. ${ }^{28}$

Automated exposure control algorithms likely account for some of the variation in dose that we noted for scans in this study, particularly CT of the abdomen (see Figure 2). As abdominal fat is quite variable between patients, these types of studies had wide interquartile ranges compared to simple head or thorax (PE) studies. Other sources of variation likely include differences in patient length (i.e., height), poor patient centring in the scanner, and choice of protocol (single phase versus multiphase). In addition, some radiologists may be more accepting of image noise than others and when on duty in the ED choose protocols accordingly. This study was not designed to investigate the reasons for the observed dose variation.

These dose estimates are limited by our inability to directly measure radiation dose to the patient. The effective doses reported in this study are calculated from the dose-length product reported by the scanner, multiplied by a conversion factor. The dose-length product is a value based on calibration scans performed on an inanimate device called a phantom. It depends on scanner settings, not on any true measurement of exposure to the patient being scanned. Furthermore, the effective dose calculation depends on the choice of conversion factors used. The true risk of inducing a cancer in any organ depends on the absorbed organ dose to that organ. ${ }^{2}$ The effective dose estimates are therefore a crude measure of risk at best. ${ }^{29}$ Even if the true organ doses are known, calculation of risk is complex as the age-dependent radiation risk to each organ is different. ${ }^{30}$ Furthermore, such calculation assumes linearity of risk at low radiation doses. This concept, the linear no-threshold model, is accepted by most scientific bodies, although there is debate. ${ }^{31-33}$

Published estimates of radiation risk from CT scanning vary. For instance, based on median doses in the year 2000, Brenner and Hall estimated the lifetime attributable risk of cancer death from an abdominal CT scan to be $0.07 \%$ ( 1 in 1,425$)$ for a 15 year-old but less than $0.02 \%$ ( 1 in 5,000$)$ for a 35 -yearold. ${ }^{2}$ Smith-Bindman and colleagues estimated the lifetime risk of developing cancer (not death) from a routine contrast-enhanced abdominal CT scan to be 1 in 470 for a 20 -year-old woman, 1 in 620 for a 20 -yearold man, 1 in 870 for a 40-year-old woman, and 1 in 942 for a 40-year-old man and noted significant variation in dose (and risk) among patients and institutions. ${ }^{6}$ The risk from radiation exposure is higher for women and lower with increasing age at exposure. ${ }^{34}$ As this study demonstrates, dose varies considerably from patient to patient. There are also inherent uncertainties in deriving radiation risk models from epidemiologic data. ${ }^{35}$ It is challenging for a clinician to make a precise estimate of the increased risk of cancer attributable to a single CT scan for an individual patient. We argue that it is important for clinicians to understand the relative doses of the CT studies they do order and the possible variations in dose. 
For clinicians, the decision to order a CT scan should be based on a consideration of the probability that the test will change management versus the probability that the test will cause harm. To weigh these issues, emergency physicians should have some knowledge of the dose and dose variation associated with the CT studies they order, but the available literature suggests that they do not. ${ }^{7}$ Unfortunately, without understanding dose, it is unlikely that emergency physicians will be able to understand the potential risk of carcinogenesis.

Even as CT techniques and technology improve and doses are constrained, the biggest factor affecting risk to the patient is the patient's age. Younger patients are more sensitive to the carcinogenic effects of radiation and have the greatest relative risk increase in cancer after exposure. ${ }^{34}$ Emergency physicians would be advised to minimize CT scanning in pediatric and young adult patients.

Although our study gives an overview of CT ordering practice at two academic EDs, within one hospital system in Canada, there are some limitations to our search strategy. We searched for scans ordered by emergency physicians. Trauma patients were underrepresented because most trauma team leaders at our institution are surgeons. Similarly, CT scans ordered by consultants would not be included, so we likely underestimated the total rate of scanning in our ED patients. Conversely, a percentage of scans were likely misattributed in the PACS database, when patients were under the care of consultants but physically still present in the ED. Nonetheless, these studies would likely represent only a small percentage of our ED population, and we do not believe that they would significantly change our dose estimates. Finally, the rate of CT scanning we observed might be greater than the rates of CT use in Canadian community EDs.

\section{CONCLUSION}

In two large, academic Canadian EDs, one in seven patients underwent CT scanning. This suggests that Canadian academic emergency physicians are high users of CT. The most common types of CT scans ordered by emergency physicians are simple (unenhanced) scans of the head, thorax (PE protocol), and abdomen for abdominal or flank pain. Doses noted in this study were somewhat higher than those in earlier studies using older CT technology. There is also considerable dose variation between studies. With increasing use of CT technology, particularly among younger patients, it is incumbent on clinicians to have some knowledge of the radiation doses associated with $\mathrm{CT}$ studies they order because of the potential cancer risk.

Acknowledgements: We thank Angela Marcantonio, My-Linh Tran, Sheryl Domingo, and Cathy Clement from the Ottawa Hospital Research Institute and Dr. Blair MacDonald of the Department of Diagnostic Imaging, University of Ottawa, for their assistance.

Competing interests: This study was funded by a research grant from the Department of Emergency Medicine, University of Ottawa.

\section{REFERENCES}

1. Kocher K, Meurer W, Fazel R, et al. National trends in use of computed tomography in the emergency department. Ann Emerg Med 2011;58:452-62.

2. Brenner D, Hall E. Computed tomography — an increasing source of radiation exposure. $N$ Engl 7 Med 2007;357:227784, doi:10.1056/NEJMra072149.

3. Berrington de González A, Mahesh M, Kim K-P, et al. Projected cancer risks from computed tomographic scans performed in the United States in 2007. Arch Intern Med 2009;169:2071-7, doi:10.1001/archinternmed.2009.440.

4. Krille L, Hammer G, Merzenich H, et al. Systematic review on physician's knowledge about radiation doses and radiation risks of computed tomography. Eur 7 Radiol 2010;76:3641, doi:10.1016/j.ejrad.2010.08.025.

5. Shrimpton $P$, Hillier M, Lewis $M$, et al. National survey of doses from CT in the UK: 2003. Br 7 Radiol 2006;79:968-80, doi:10.1259/bjr/93277434.

6. Smith-Bindman R, Lipson J, Marcus R, et al. Radiation dose associated with common computed tomography examinations and the associated lifetime attributable risk of cancer. Arch Intern Med 2009;169:2078-86, doi:10.1001/archinternmed. 2009.427

7. Lee C, Haims A, Monico E, et al. Diagnostic CT scans: assessment of patient, physician, and radiologist awareness of radiation dose and possible risks. Radiology 2004;231:393-8, doi:10.1148/radiol.2312030767.

8. Huda W, Ogden K, Khorasani M. Converting dose-length product to effective dose at CT. Radiology 2008;248:9951003, doi:10.1148/radiol.2483071964.

9. Huda W, Mettler F. Volume CT dose index and dose-length product displayed during CT: what good are they? Radiology 2011;258:236-42, doi:10.1148/radiol.10100297.

10. Lee J, Kirschner J, Pawa S, et al. Computed tomography use in the adult emergency department of an academic urban hospital from 2001 to 2007. Ann Emerg Med 2010;56:5916.e1, doi:10.1016/j.annemergmed.2010.05.027.

11. Raja A, Walls R, Schuur J. Decreasing use of high-cost imaging: the danger of utilization-based performance 
measures. Ann Emerg Med 2010;56:597-9, doi:10.1016/ j.annemergmed.2010.09.013.

12. Sodickson A, Baeyens PF, Andriole KP, et al. Recurrent CT, cumulative radiation exposure, and associated radiationinduced cancer risks from CT of adults. Radiology 2009;251: 175-84, doi:10.1148/radiol.2511081296.

13. Fazel R, Krumholz H, Wang Y, et al. Exposure to low-dose ionizing radiation from medical imaging procedures. $N \mathrm{Engl}$ 7 Med 2009;361:849-57, doi:10.1056/NEJMoa0901249.

14. Bendeck S, Nino-Murcia M, Berry G, et al. Imaging for suspected appendicitis: negative appendectomy and perforation rates. Radiology 2002;225:131-6, doi:10.1148/radiol. 2251011780.

15. Frei S, Bond $W$, Bazuro R, et al. Appendicitis outcomes with increasing computed tomographic scanning. Am 7 Emerg Med 2008;26:39-44, doi:10.1016/j.ajem.2007.06.027.

16. Coursey C, Nelson R, Patel M, et al. Making the diagnosis of acute appendicitis: do more preoperative CT scans mean fewer negative appendectomies? A 10-year study. Radiology 2010;254:460-8, doi:10.1148/radiol.09082298.

17. Broder J, Bowen J, Lohr J, et al. Cumulative CT exposures in emergency department patients evaluated for suspected renal colic. 7 Emerg Med 2007;33:161-8, doi:10.1016/j. jemermed.2006.12.035.

18. Katz SI, Saluja S, Brink JA, et al. Radiation dose associated with unenhanced CT for suspected renal colic: impact of repetitive studies. A7R Am $\mathcal{F}$ Roentgenol 2006;186:1120-4, doi:10.2214/AJR.04.1838.

19. Goldstein NM, Kollef MH, Ward S, et al. The impact of the introduction of a rapid D-dimer assay on the diagnostic evaluation of suspected pulmonary embolism. Arch Intern Med 2001;161:567-71, doi:10.1001/archinte.161.4.567.

20. Pollack CV. Clinical characteristics, management, and outcomes of patients diagnosed with acute pulmonary embolism in the emergency department. $7 \mathrm{Am}$ Coll Cardiol 2011;57:700-6, doi:10.1016/j.jacc.2010.05.071.

21. Venkatesh A, Kline J, Courtney D, et al. Evaluation of pulmonary embolism in the emergency department and consistency with a national quality measure. Arch Intern Med. [Epub ahead of print 2012 June 4]

22. Kline JA, Courtney DM, Beam DM, et al. Incidence and predictors of repeated computed tomographic pulmonary angiography in emergency department patients. Ann Emerg Med 2009;54:41-8, doi:10.1016/j.annemergmed.2008.08.015.

23. Anderson DR, Kahn SR, Rodger MA, et al. Computed tomographic pulmonary angiography vs ventilation-perfusion lung scanning in patients with suspected pulmonary embolism: a randomized controlled trial. FAMA 2007;298:2743-53, doi: $\underline{10.1001 / \text { jama.298.23.2743. }}$
24. Hadley J, Agola J, Wong P. Potential impact of the American College of Radiology appropriateness criteria on CT for trauma. A7R Am $\mathcal{f}$ Roentgenol 2006;186:937-42, doi:10.2214/AJR.05.0041.

25. Stiell IG, Clement CM, Grimshaw JM, et al. A prospective cluster-randomized trial to implement the Canadian CT Head Rule in emergency departments. CMAJ 2010;182: 1527-32, doi:10.1503/cmaj.091974.

26. Macgregor DM, McKie L. CT or not CT - that is the question. Whether 'tis better to evaluate clinically and $\mathrm{x}$-ray than to undertake a CT head scan! Emerg Med 7 2004;22: 541-3, doi:10.1136/emj.2004.017160.

27. Bankier A, Kressel H. Through the Looking Glass revisited: the need for more meaning and less drama in the reporting of dose and dose reduction in CT. Radiology 2012;265:4-8, doi:10.1148/radiol.12121145.

28. Israel G, Cicchiello L, Brink J, et al. Patient size and radiation exposure in thoracic, pelvic, and abdominal ct examinations performed with automatic exposure control. A7R Am 7 Roentgenol 2010;195:1342-6, doi:10.2214/AJR.09.3331.

29. Brenner D. Is it time to retire the CTDI for CT quality assurance and dose optimization? Med Phys 2005;32:3225-6, doi:10.1118/1.2040747.

30. Brenner D, Elliston C, Hall E, et al. Estimated risks of radiation-induced fatal cancer from pediatric CT. $A 7 R$ Am 7 Roentgenol 2001;176:289-96, doi:10.2214/ajr.176.2. 1760289.

31. Brenner D, Doll R, Goodhead D, et al. Cancer risks attributable to low doses of ionizing radiation: assessing what we really know. Proc Natl Acad Sci U S A 2003;100:13761-6, doi:10.1073/pnas.2235592100.

32. Little M, Wakeford R, Tawn E, et al. Risks associated with low doses and low dose rates of ionizing radiation: why linearity may be (almost) the best we can do. Radiology 2009; 251:6-12, doi:10.1148/radiol.2511081686.

33. Tubiana M, Feinendegen L, Yang C, et al. The linear nothreshold relationship is inconsistent with radiation biologic and experimental data. Radiology 2009;251:13-22, doi:10. 1148/radiol.2511080671.

34. Preston DL, Ron E, Tokuoaka S, et al. Solid cancer incidence in atomic bomb survivors: 1958-1998. Radiat Res 2007;168:1-64, doi:10.1667/RR0763.1.

35. Committee to Assess Health Risks from Exposure to Low Levels of Ionizing Radiation, National Research Council. Estimating Cancer Risk. In: Health Risks from Exposure to Low Levels of Ionizing Radiation: BEIR VII Phase 2. Washington (DC): The National Academies Press; 2006. p. 267-312. 